The Astrophysical Journal, 474:L19-L22, 1997 January 1

(C) 1997. The American Astronomical Society. All rights reserved. Printed in U.S.A.

\title{
DYNAMICAL CORRELATIONS FOR GLOBULAR CLUSTERS IN M311,2
}

\author{
S. G. Djorgovski, ${ }^{3,4}$ R. R. Gal, ${ }^{3}$ J. K. McCarthy, ${ }^{3}$ J. G. Cohen, ${ }^{3}$ R. R. de Carvalho, ${ }^{3,5}$ G. Meylan, ${ }^{6}$ \\ O. Bendinelli, ${ }^{7}$ AND G. PARMEGGiani ${ }^{8}$ \\ Received 1996 August 20; accepted 1996 October 16
}

\begin{abstract}
We present internal velocity dispersion measurements for a set of 21 globular clusters in the Andromeda galaxy (M31). We combine them with structural and photometric cluster parameters measured earlier with the Hubble Space Telescope and from the ground to explore correlations of cluster properties and to compare them with the equivalent correlations for the Galactic globular clusters. We find that the M31 globulars follow the same correlations between velocity dispersion and luminosity, central, and average surface brightness, as do their Galactic counterparts. This suggests a common physical origin for these correlations. They may be produced by the same astrophysical conditions and processes operating at the epoch of globular cluster formation in both galaxies. The very existence of these excellent correlations, and their quantitative form as scaling laws, represent challenges and constraints for theories of globular cluster formation. Preliminary estimates of the cluster $M / L$ ratios show correlations with the cluster metallicity, in the sense of more metal-rich clusters having lower $M / L$, particularly in the near-infrared. At a given metallicity, there is no detectable systematic difference between the M31 globulars and their Galactic counterparts, which suggests a great similarity of their stellar populations. The observed scatter around these trends is comparable to the expected errors, which implies a small intrinsic scatter and thus which leaves little room for possible variations in the cluster age or stellar IMF at a given metallicity. Subject headings: galaxies: individual: M31 — galaxies: star clusters — globular clusters: general
\end{abstract}

\section{INTRODUCTION}

Globular clusters (GCs) contain unique information about old stellar populations and their formation. Comparison of GC systems in different galaxies can help discriminate between different formation mechanisms of clusters themselves and of their parent galaxies. Detailed correlations of GC properties are particularly useful for this task but so far have been limited to the Galactic GC system (Djorgovski \& Meylan 1994, Bellazzini et al. 1996, Djorgovski 1995, and references therein). To date, studies of GC systems in other galaxies have been largely limited to various photometric data (see, e.g., a review by Harris 1991), with limited spectroscopic studies (see, e.g., Peterson 1988; Huchra, Brodie, \& Kent 1991) for M31 and a few other nearby systems. Reliable internal dynamical measurements have been obtained only for GCs in dwarf satellites of our Galaxy (see, e.g., Dubath, Meylan, \& Mayor 1992; Dubath, Mayor, \& Meylan 1993; Dubath, Meylan, \& Mayor 1996).

Among the many correlations seen among the properties of Galactic GCs, those involving velocity dispersions (VDs) are particularly interesting. While their origin is still not understood, they may reflect conditions of cluster formation rather than their subsequent dynamical evolution (Djorgovski \& Meylan 1994). VDs are also necessary in order to compute the

\footnotetext{
${ }^{1}$ Based in part on observations obtained at the W. M. Keck Observatory, which is operated jointly by the California Institute of Technology and the University of California.

${ }^{2}$ Based in part on observations obtained with the NASA/ESA Hubble Space Telescope, operated by the Space Telescope Science Institute, which is operated by AURA, Inc., under NASA contract NAS 5-26555.

3 Palomar Observatory, MS 105-24, Caltech, Pasadena, CA 91125.

${ }^{4}$ Presidential Young Investigator.

5 On leave from Observatorio Nacional, CNPq, Rio de Janeiro, Brasil.

${ }^{6}$ European Southern Observatory, D-85748 Garching, Germany.

7 Dipartimento di Astronomia, Università di Bologna, I-40126 Bologna, Italy.

${ }_{8}$ Osservatorio Astronomico, Via Zamboni 33, I-40126 Bologna, Italy.
}

$M / L$ ratios, one of the fundamental quantities describing stellar populations.

We present here some initial results from a study of GCs in M31, using new measurements of their VDs, combined with a variety of photometric data obtained earlier with the Hubble Space Telescope (HST) and from the ground. Previous work on the M31 GC system has been reviewed by Fusi Pecci et al. (1993), Huchra (1993), Tripicco (1993), and Cohen (1993).

\section{OBSERVATIONS AND DATA REDUCTIONS}

Data were obtained using the HIRES spectrograph (Vogt et al. 1994) at the W. M. Keck 10 m telescope on UT 1994 August 15-16, October 5-7, and 1995 August 6-7. Total exposure times were in the range of $1-2 \mathrm{hr}$. We used a 1". $15 \times 7$ ". 0 slit, giving a FWHM spectral resolution $R=\lambda / \Delta \lambda=35,000$. The wavelength range covered was $4300 \leq \lambda \leq 6630 \AA$ in 29 echelle orders. We observed $21 \mathrm{GCs}$, selected mainly on the account of having HST images available. In general, they have luminosities comparable to or greater than the brightest Galactic GCs, which simply reflects the larger population of M31 GCs.

Data were reduced using echelle tasks in the Figaro package (McCarthy 1990). The echelle orders were extracted using an aperture typically 2."65 wide. Th-Ar arc spectra were used for wavelength calibration, giving typical residuals of $8 \mathrm{m \AA} \mathrm{rms.}$ The analysis was performed independently on each of the spectral orders for all objects. We used the Fourier fitting technique of Franx, Illingworth, \& Heckman (1989). Standard stars ranging in spectral type were tested as templates; HR 8684 ( $\mu$ Peg; type G8 III; $v \sin i=7 \mathrm{~km} \mathrm{~s}^{-1}$ ) provided the most stable results and was adopted as our template. All GC spectra were first cross-correlated with the template in order to find and remove the radial velocity differences. VDs were then measured for each order of each GC, and these measurements were then averaged. Table 1 lists the mean observed values (in 
TABLE 1

Velocity Dispersion Measurements

\begin{tabular}{|c|c|c|c|c|}
\hline Cluster & $\sigma_{\mathrm{obs}}$ & RMS & $N_{\text {ord }}$ & Error \\
\hline G1... & 25.06 & 1.66 & 27 & 0.32 \\
\hline $\mathrm{G} 2 \ldots$ & 9.70 & 1.43 & 26 & 0.29 \\
\hline G58... & 11.56 & 0.58 & 27 & 0.11 \\
\hline$\ldots \ldots \ldots$ & 16.15 & 2.20 & 24 & 0.45 \\
\hline G73... & 14.27 & 1.22 & 27 & 0.24 \\
\hline$\ldots \ldots \ldots$ & 25.46 & 4.67 & 27 & 0.90 \\
\hline G105 ........... & 9.08 & 1.86 & 23 & 0.39 \\
\hline G108 .......... & 9.82 & 0.94 & 26 & 0.18 \\
\hline $\mathrm{G} 213$. & 20.50 & 1.35 & 27 & 0.26 \\
\hline G219. & 8.11 & 1.74 & 23 & 0.36 \\
\hline $\mathrm{G} 244 \ldots \ldots \ldots \ldots$ & 13.20 & 1.24 & 27 & 0.24 \\
\hline G272 . & 17.62 & 1.24 & 27 & 0.24 \\
\hline G280 . & 25.94 & 1.99 & 27 & 0.38 \\
\hline G302 .......... & 11.92 & 1.15 & 27 & 0.22 \\
\hline G305 ......... & 12.58 & 0.77 & 27 & 0.15 \\
\hline G312 ....... & 8.15 & 1.02 & 26 & 0.20 \\
\hline G319 ..... & 10.10 & 0.82 & 27 & 0.16 \\
\hline G322 .......... & 11.49 & 1.24 & 27 & 0.24 \\
\hline $\mathrm{G} 351 \ldots \ldots \ldots \ldots$ & 8.57 & 1.74 & 15 & 0.45 \\
\hline G352 .......... & 9.52 & 0.59 & 27 & 0.11 \\
\hline Bo $289 \ldots \ldots \ldots$ & 8.43 & 2.09 & 22 & 0.44 \\
\hline
\end{tabular}

$\mathrm{km} \mathrm{s}^{-1}$ ), the rms scatter between different orders, the number of the orders used, and the final uncertainty. These data have lower relative errors than most VD measurements now available for Galactic GCs. We have five clusters in common with Peterson (1988); our measurements agree to within the quoted errors in three cases; for the remaining two, Peterson gives $\sigma \geq 40 \mathrm{~km} \mathrm{~s}^{-1}$, which we believe are overestimates.

In order to reduce the observed VDs to standard values suitable for comparisons with Galactic GCs, we estimate the aperture corrections as follows. We use King-Michie dynamical models of GCs (King 1966), as described by Meylan (1987). We integrated the projected, luminosity-weighted VD in an aperture of a given radius, expressed as a function of the cluster half-light radius, $r_{h}$. Ratios of these aperture-averaged VDs to the projected central VD as a function of radius are plotted in Figure 1 for a range of models of different concentrations, $c$. The dependence on $c$ is rather weak, and we evaluate the corrections at a "typical" value of $c=1.8$, the median for the sample of Galactic GCs with available VDs. Our rectangular measurement aperture, convolved with the

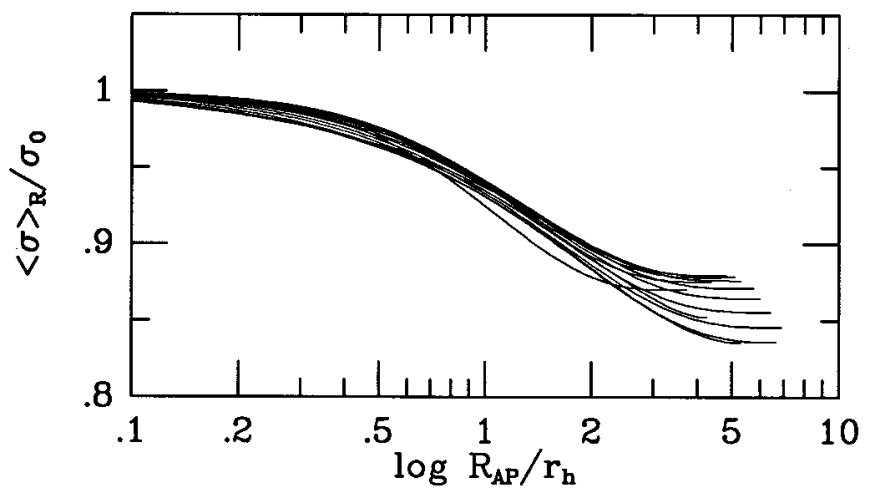

FIG. 1.-Velocity dispersion aperture corrections for King-Michie models. The ratio of the projected, luminosity-weighted velocity dispersion averaged over an aperture of a radius $R_{\mathrm{AP}},\langle\sigma\rangle_{R}$, to the projected central velocity dispersion, $\sigma_{0}$, is shown as a function of aperture radius in half-light radius $\left(r_{h}\right)$ units. Curves are plotted for a set of models with concentration parameters ranging from $c=0.89$ to 2.48 . typical seeing, generally corresponds to $\sim 2-3 r_{h}$. We thus adopted a fixed-aperture correction for all of our measurements, increasing the observed VD values by $14 \%$ to estimate the standard, projected central values, $\sigma_{0}$. The net uncertainty of this aperture correction, including the individual GC variations, is estimated to be a few percent, comparable to the random measurement errors.

We supplement these measurements with photometric and structural parameters taken from the literature. Structural parameters from $H S T$ are available for 14 objects, taken from Bendinelli et al. (1993), Fusi Pecci et al. (1994), Rich et al. (1996), and Grillmair et al. (1996). Ground-based photometry in $V$ and $K$ bands and reddening estimates are from Reed, Harris, \& Harris (1994), Bonoli et al. (1987), Battistini et al. (1987), Frogel, Persson, \& Cohen (1980), Sitko (1984), and Cohen \& Matthews (1994), and metallicities are from Huchra et al. (1991). We assume $(m-M)=24.43$, i.e., a distance of $770 \mathrm{kpc}$. As a comparison sample, we use 55 Galactic GCs for which $\sigma_{0}$ measurements are available (Pryor \& Meylan 1993) with photometric and structural parameters from Djorgovski (1993b) and infrared measurements for some of them from Aaronson et al. (1978). For both samples, central and averaged (within $r_{h}$ ) surface brightness measurements are available in the $V$ band. We used the reddening-corrected $(V-K)$ colors to derive the corresponding quantities in the $K$ band.

\section{RESULTS AND DISCUSSION}

Correlations between the central projected VD $\left(\sigma_{0}\right)$, and the absolute magnitude $(M)$, central $\left(\mu_{0}\right)$, and half-lightaveraged $\left(\langle\mu\rangle_{h}\right)$ surface brightness in both $V$ and $K$ bands are shown in Figure 2. The M31 GCs smoothly continue the trends seen in the Galactic sample, extending them to higher luminosities. The two samples show the same slopes and no detectable differences in intercepts of these correlations. This is one of our principal results, which suggests that these correlations are common to GC systems in different galaxies, perhaps reflecting some universal physics of their formation or evolution. The observed scatter in these correlations is comparable to what may be expected from the measurement errors and uncertainties in reddenings, relative distances, and our aperture corrections. Curiously, the scatter is lower for the M31 GC data, perhaps owing to the smaller uncertainties in their relative distances and reddenings. This is also the first time that such correlations have been clearly demonstrated for a GC system in another galaxy.

For all of these correlations, we performed least-squares fits that allow for errors in both coordinates, giving an equal statistical weight to all data points. Fits were performed for the M31 and the Galactic samples separately and for the combined sample. In general, there is a good agreement between different samples and the two bandpasses used. These correlations correspond to a set of scaling laws, listed in Table 2. (Here we use the symbols $L$ and $I$ to denote luminosities and surface brightness in linear flux units, rather than magnitudes.) The derived power-law exponents are in a good agreement with the previous evaluations for Galactic GCs (Djorgovski \& Meylan 1994).

The origin of these correlations is unclear. They are unlikely to be produced by the secular dynamical evolution of GCs. Central VD changes only weakly during core collapse, corresponding to a scaling law $\sigma_{0} \sim I_{0}^{0.09}$, and cluster half-light parameters are almost unaffected. At least in the Galactic GC 

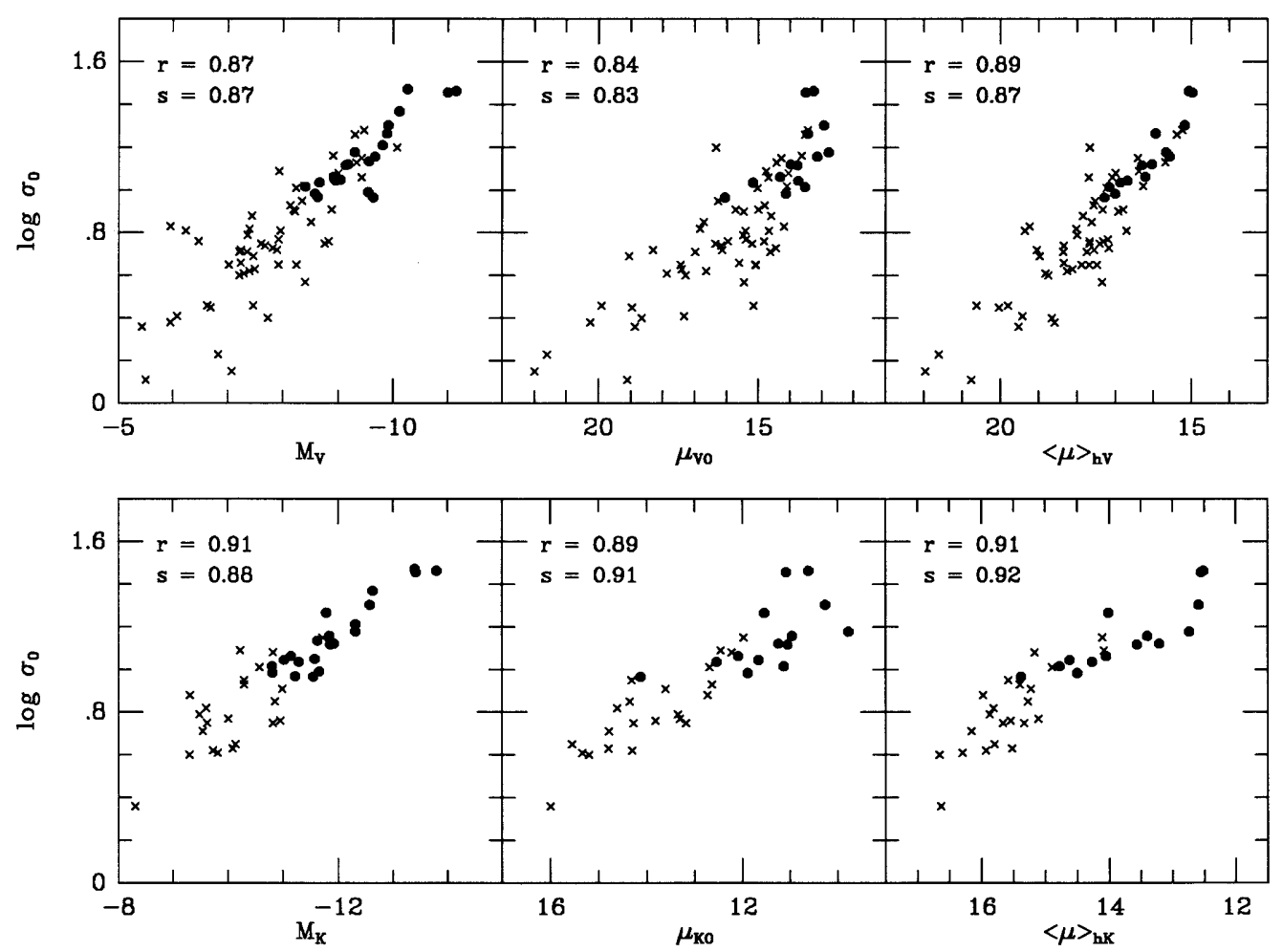

FIG. 2.-Velocity dispersion correlations for the M31 GC sample ( filled circles) and the Galactic GC sample (crosses). Projected central velocity dispersion ( $\sigma_{0}$, in $\mathrm{km} \mathrm{s}^{-1}$ ) is plotted against the absolute magnitude $(M)$, central $\left(\mu_{0}\right)$, and half-light averaged $\left(\langle\mu\rangle_{h}\right)$ surface brightness. The top row shows the measurements in the $V$ band, and the bottom row, in the $K$ band. Pearson linear regression $(r)$ and Spearman rank $(s)$ correlation coefficients are shown in each panel.

sample, VDs do not correlate well with quantities that seem to reflect dynamical evolution of clusters. It is more likely that the VD correlations reflect the physical conditions and mechanisms close to the epoch of GC formation, e.g., the properties of their progenitor systems or the effects of early stellar evolution and mass loss. Speculations about possible mechanisms that could generate these correlations have been discussed by Djorgovski (1993a) and Djorgovski \& Meylan (1994), but their understanding remains both a challenge and a significant observational constraint for theories of GC formation and evolution. These correlations are very different from the corresponding ones for dwarf spheroidals or for elliptical galaxies (Djorgovski 1993a).

The next step would be to explore bivariate correlations of M31 GC properties, as was done for the Galactic sample by Djorgovski (1995). This requires a larger sample of objects with HST surface photometry and will be addressed in a future paper.

Pending a more detailed dynamical modeling of individual clusters, which is beyond the scope of this Letter, we can also estimate their approximate $M / L$ ratios through the relation

TABLE 2

SCAling LAw Slopes $\alpha$ FOR Relations $X \sim \sigma^{\alpha}$

\begin{tabular}{|c|c|c|c|}
\hline$X$ & M31 & Galactic & Combined \\
\hline$L_{V}$ & $1.9 \pm 0.15$ & $1.7 \pm 0.3$ & $1.7 \pm 0.15$ \\
\hline & $2.1 \pm 0.15$ & $1.8 \pm 0.35$ & $2.0 \pm 0.15$ \\
\hline$I_{0 V} \ldots \ldots \ldots \ldots$ & $2.6 \pm 0.8$ & $3.3 \pm 0.4$ & $3.0 \pm 0.4$ \\
\hline$I_{0 K} \ldots \ldots \ldots \ldots$ & $2.4 \pm 1.0$ & $2.6 \pm 0.2$ & $2.8 \pm 0.2$ \\
\hline$\langle I\rangle_{h V} \ldots$ & $2.0 \pm 0.1$ & $2.2 \pm 0.2$ & $2.1 \pm 0.15$ \\
\hline$\langle I\rangle_{h K}$ & $2.4 \pm 0.3$ & $1.5 \pm 0.15$ & $2.0 \pm 0.15$ \\
\hline
\end{tabular}

$M / L \approx k \sigma^{2} r_{h} / L$, where $k$ is a parameter that depends on the cluster structure. In order to minimize possible systematic errors for our comparison, here we restrict the Galactic GC sample to clusters having a comparable range of luminosity, $\sigma_{0}$, and $r_{h}$ to our M31 GC sample, viz., $M_{V}<-8, \log \sigma_{0}>0.9$, and $0.2<\log r_{h}<0.7$.

Figure 3 shows the dependence of these preliminary $M / L$

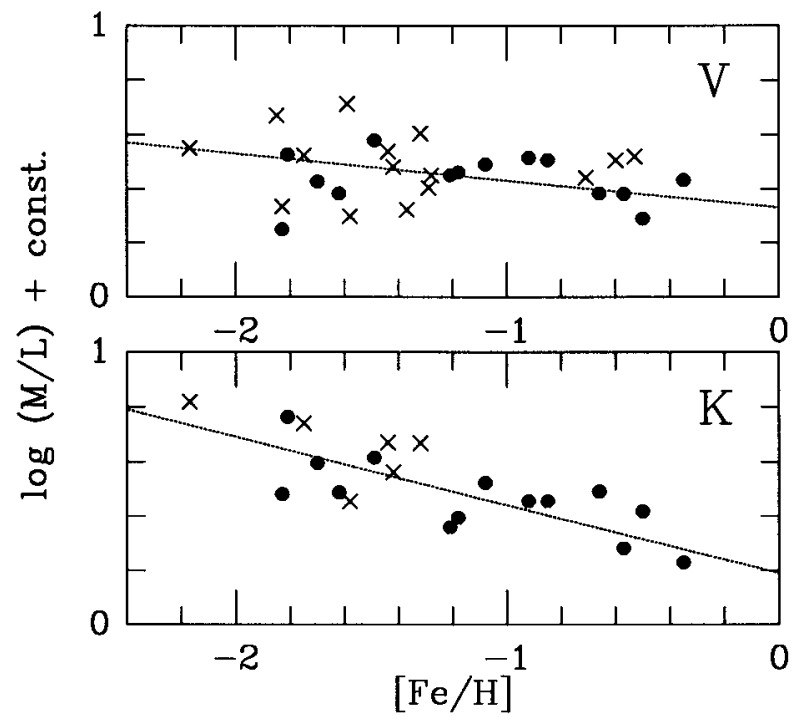

Fig. 3.-Dependence of the apparent $M / L$ ratios (to within an arbitrary constant) in the $V$ (top) and the $K$ (bottom) bands, on the cluster metallicity, $[\mathrm{Fe} / \mathrm{H}]$. M31 GCs are plotted as solid dots, and a comparison Galactic GC sample, as crosses. The dotted lines show the best fits for the M31 GC sample. 
ratios on the metallicity. There is an obvious trend in the $K$ band, with the best-fit slope for the M31 GC sample of $\Delta\left[\log (M / L)_{K}\right] / \Delta[\mathrm{Fe} / \mathrm{H}]=-0.25 \pm 0.05$ and a weaker trend in the $V$ band, with the slope of $-0.1 \pm 0.1$. These slopes do not change significantly if Galactic GCs are added to the fits. Indeed, we find no detectable difference in the median intercepts for the M31 and the Galactic samples. This by itself is an interesting result: at a given metallicity, Galactic and M31 GCs have the same $M / L$ ratios to within a few percent and presumably have similar stellar populations. Using a completely independent approach, Cohen \& Freeman (1991) have already suggested that the average $M / L$ ratios are broadly similar between the two GC systems, but the present result is quantitatively much stronger.

The physical cause of these trends may be in the metallicity dependence of the red giant branch luminosities, particularly in the $K$ band, e.g., more luminous at higher metallicities, and/or a possible metallicity dependence of the stellar IMF, e.g., steeper slope at lower metallicities, an effect suggested previously for the Galactic GCs (McClure et al. 1986; Djor- govski, Piotto, \& Capaccioli 1993). Both samples show a rms scatter of only about 0.11 in $\log M / L$, i.e., $<30 \%$ in $M / L$, around these fits. Most or all of that can be accounted for by the measurement errors and various possible systematics, which leaves little room for possible intrinsic differences, owing, e.g., to a spread in GC ages or the stellar IMF. A more detailed modeling of these effects should lead to interesting constraints on the possible age or IMF differences, both within the M31 GC system, and between it and the Galactic GC system.

We acknowledge expert help of the staff of the W. M. Keck Observatory, particularly T. Chelminiak, and the work of many collaborators on the HST M31 GC team, especially F. Fusi Pecci. G1 data were kindly communicated by R. M. Rich and D. Neill. This work was supported in part by the NSF PYI award AST-9157412 to S. G. D.; NASA grants GO-2583, 3726, and 5420 from STScI; and an NSF graduate fellowship to R. R. G.
Aaronson, M., Cohen, J. G., Mould, J., \& Malkan, M. 1978, ApJ, 223, 824

Battistini, P., Bonoli, F., Braccesi, A., Federici, L., Fusi Pecci, F., Marano, B., \& Börngen, F. 1987, A\&ASS, 67, 447

Bellazzini, M., Vesperini, E., Ferraro, F., \& Fusi Pecci, F. 1996, MNRAS, 279, 337

Bendinelli, O., et al. 1993, ApJ, 409, L17

Bonoli, F., Delpino, F., Federici, L., \& Fusi Pecci, F. 1987, A\&A, 185, 25

Cohen, J. G. 1993, in ASP Conf. Proc. 48, The Globular Cluster-Galaxy Connection, ed. G. Smith \& J. Brodie (San Francisco: ASP), 438

Cohen, J. G., \& Freeman, K. L. 1991, AJ, 101, 483

Cohen, J. G., \& Matthews, K. 1994, AJ, 108, 128

Djorgovski, S. 1993a, in ASP Conf. Proc. 48, The Globular Cluster-Galaxy Connection, ed. G. Smith \& J. Brodie (San Francisco: ASP), 496 - 1993b, in ASP Conf. Proc. 50, Structure and Dynamics of Globular

Clusters, ed. S. Djorgovski \& G. Meylan (San Francisco: ASP), 373 1995, ApJ, 438, L29

Djorgovski, S., \& Meylan, G. 1994, AJ, 108, 1292

Djorgovski, S., Piotto, G., \& Capaccioli, M. 1993, AJ, 105, 2148

Dubath, P., Mayor, M., \& Meylan, G. 1993, in ASP Conf. Proc. 48, The Globular Cluster-Galaxy Connection, ed. G. Smith \& J. Brodie (San Francisco: ASP), 557

Dubath, P., Meylan, G., \& Mayor, M. 1992, ApJ, 400, 510

. 1996, A\&A, submitted

Franx, M., Illingworth, G., \& Heckman, T. 1989, ApJ, 344, 613

Frogel, J., Persson, S. E., \& Cohen, J. G. 1980, ApJ, 240, 785

Fusi Pecci, F., Cacciari, C., Federici, L., \& Pasquali, A. 1993, in ASP Conf. Proc. 48, The Globular Cluster-Galaxy Connection, ed. G. Smith \& J. Brodie (San Francisco: ASP), 410

\section{REFERENCES}

Fusi Pecci, F., et al. 1994, A\&A, 284, 349

Grillmair, C., Ajhar, E., Faber, S., Baum, W., Holtzman, J., Lauer, T., Lynds, C. R., \& O'Neil, E. 1996, AJ, 111, 2293

Harris, W. 1991, ARA\&A, 29, 543

Huchra, J. 1993, in ASP Conf. Proc. 48, The Globular Cluster-Galaxy Connection, ed. G. Smith \& J. Brodie (San Francisco: ASP), 420

Huchra, J., Brodie, J., \& Kent, S. 1991, ApJ, 370, 495

King, I. R. 1966, AJ, 71, 64

McCarthy, J. K. 1990, in Proc. 2nd ESO/ST-ECF Data Analysis Workshop, ed. D. Baade \& P. J. Grosbol (Garching: European Southern Observatory), 119 McClure, R. D., et al. 1986, ApJ, 307, L49

Meylan, G. 1987, A\&A, 184, 144

Peterson, R. 1988, in Dynamics of Dense Stellar Systems, ed. D. Merritt (Cambridge: Cambridge Univ. Press), 161

Pryor, C., \& Meylan, G. 1993, in ASP Conf. Proc. 50, Structure and Dynamics of Globular Clusters, ed. S. Djorgovski \& G. Meylan (San Francisco: ASP), 357

Reed, L., Harris, G., \& Harris, W. 1994, AJ, 107, 555

Rich, R. M., Mighell, K., Freedman, W., \& Neill, J. D. 1996, AJ, 111, 768

Sitko, M. 1984, ApJ, 286, 209

Tripicco, M. 1993, in ASP Conf. Proc. 48, The Globular Cluster-Galaxy

Connection, ed. G. Smith \& J. Brodie (San Francisco: ASP), 432

Vogt, S., et al. 1994, Proc. SPIE, 2198, 362 